\title{
Microbial community and diversity in the feces of Sichuan takin (Budorcas taxicolor tibetana) as revealed by Illumina Miseq sequencing and quantitative real-time PCR
}

Hui Zhu ${ }^{1,2,3 \dagger}$, Dong Zeng ${ }^{1,2 \dagger}$, Ning Wang ${ }^{3,4}$, Li-li Niu${ }^{5}$, Yi Zhou ${ }^{1,2}$, Yan Zeng ${ }^{1,2}$ and Xue-qin $\mathrm{Ni}^{1,2^{*}}$

\begin{abstract}
The Sichuan takin (Budorcas taxicolor tibetana) is a rare and endangered ruminant distributed in the eastern Himalayas. However, little information is available regarding the intestinal microbiota of the takin. In this study, Illumina Miseq platform targeting the $\mathrm{V} 4$ region of the $16 \mathrm{~S}$ rRNA was employed to characterize microbial community and diversity in the feces of wild $(n=6)$ and captive takins $(n=6)$. The takin exhibited an intestinal microbiota dominated by three phyla: Firmicutes (57.4\%), Bacteroidetes (24.2\%) and Proteobacteria (12.3\%). At family/genus level, Ruminococcaceae, Bacteroidaceae, Acinetobacter, Clostridium, Lachnospiraceae, Rikenellaceae, Bacillus, Comamonas and Spirochaetaceae were dominant. Distinctive microbiotas between wild and captive takins were observed based on microbial community structure, captive takins having significantly higher community diversity. Quantitative real-time PCR were also utilized to monitor predominant bacteria in three Sichuan takin individuals housed in Chengdu Zoo over a halfyear period, which showed that microbial communities of the three takins were relatively similar to each other and stable during our study period. Our results suggested that diet was a major driver for shaping microbial community composition.
\end{abstract}

Keywords: Budorcas taxicolor tibetana, Fecal microbiota, Illumina Miseq sequencing, Quantitative real-time PCR

\section{Introduction}

The microbial populations that reside in the digestive tract of animals are diverse and numerous. Generally, bacteria comprise $40-45 \%$ of fecal material on a dry weight basis with populations often exceeding $10^{11} \mathrm{col}-$ ony-forming unit (CFU) per gram feces (Eckburg et al. 2005; Stephe and Cummings 1980). The bacteria of the animal gastrointestinal tract constitutes a complex ecosystem which is involved in host physiology, ranging from the structure and functions of the digestive system and the innate and adaptive immune systems, to host energy metabolism (Macfarlane and Macfarlane 2004). Conversely, the composition of the intestinal microbiota

\footnotetext{
*Correspondence: xueqinni@foxmail.com

${ }^{\dagger}$ Hui Zhu and Dong Zeng contributed equally to this work.

${ }^{1}$ Animal Microecology Institute, College of Veterinary, Sichuan

Agricultural University, Chengdu 611130, China

Full list of author information is available at the end of the article
}

is also influenced by diet, social interactions, antibiotic use, host anatomy and phylogeny (Russell and Rychlik 2001; Ley et al. 2008b). Although there is a profound relationship between intestinal bacteria and animal health, this ecosystem of many herbivores remains incompletely characterized and its diversity poorly defined.

The takin (Budorcas taxicolor), also called cattle chamois or gnu goat, is a rare and endangered species distributed in the eastern Himalayas, which is considered a flag species for wildlife conservation. There are four subspecies of the takin, namely the Mishmi takin (B. taxicolor taxicolor), the golden takin (B. taxicolor bedfordi), the Sichuan takin (B. taxicolor tibetana), and the Bhutan takin (B. taxicolor whitei). The Sichuan takin and golden takin are endemic subspecies of China. The body size of the takin is similar to the gaur (Bos gaurus) and the wild yak (Bos grunniens), but their external characteristics of digestive system are rather similar to sheep according 
to anatomical records in Chengdu Zoo. As a large ruminant, the takin has a specialized digestive system with a four chambered stomach and a relatively long lower tract (small and large intestine), which are adapted to forage rich in plant cell wall. In systematic zoology, the takin belongs to Bovidae Ovibovinae with its close relative, the muskoxen (Ovibos moschatus). However, recent mitochondrial researches suggested that takins were more closely related to various sheep and that similarities between takins and muskoxen might be attributed to convergent evolution (Groves and Shields 1997).

Wild Sichuan takins generally are found on forested slopes at $1500-4000 \mathrm{~m}$ in elevation. They feed on many kinds of alpine deciduous plants and evergreens, which varies with season and local food availability. This mainly includes the leaves and shoots of bamboo, tough leaves of evergreen rhododendrons and oaks, willow and pine bark, and a variety of new-growth leaves and herbs (Wu and $\mathrm{Hu} 2001$ ).

The aim of the present study was to characterize the intestinal microbiota of the takin and investigate how diet and environmental factors affect the intestinal microbiota. Since intestinal microbiota have not yet been studied precisely in takin feces to our knowledge, our assays will contribute to enhanced knowledge of the takin intestinal bacteria, for further use in updating the current knowledge of the putative role of intestinal bacteria in health and disease.

\section{Materials and methods Sampling}

Fresh feces were collected from 6 wild takins in Labahe Nature Reserve $\left(30^{\circ} 18^{\prime} \mathrm{N} 102^{\circ} 27^{\prime} \mathrm{E}\right)$ and 6 captive takins in Chengdu Zoo (Chengdu, Sichuan, China) from late September to October. An adult female takin (subject A, 13-year-old), an adult male takin (subject B, 8-year-old), and a young male takin (subject $\mathrm{C}$, 3-year-old) housed separately at the Chengdu Zoo were also monitored over a half-year period. In January 5, March 2, April 27 and June 19, fecal samples were collected from the three takins respectively. The Sichuan takins housed in Chengdu Zoo were fed a constant and balanced diet, which refer to standards for maintenance requirement of cattle and sheep. Their daily diet of captive animals consisted of 20-25 kg green fodder (perennial ryegrass or Sudangrass), $2.5 \mathrm{~kg}$ alfalfa bale, $2 \mathrm{~kg}$ chopped alfalfa hay and $2 \mathrm{~kg}$ concentrate. The composition of the concentrate was $39 \%$ corn, $13 \%$ rice bran, $2 \%$ fish meal, $4 \%$ wheat middling, $15 \%$ wheat bran, $8 \%$ soybean meal, $12 \%$ cracked soybean, $1.5 \%$ hydrolyzed feather meal, $1.5 \%$ calcium carbonate, $2 \%$ dicalcium phosphate, $0.8 \%$ sodium chloride and $1.2 \%$ microelements and vitamins. For the reason of animal conservation, sampling work was accomplished by specific zoo keepers in Chengdu or staff at the reserve. Fresh fecal samples were immediately collected upon defecation around feeding time or in the process of feeding salts by park rangers. Samples were sealed in sterile plastic bags, transported to the laboratory in liquid nitrogen and then stored at $-70^{\circ} \mathrm{C}$ until analysis.

\section{Ethics statement}

All animals were handled in strict accordance with the animal protection law of the People's Republic of China (a draft animal protection law was released on September 18, 2009). All procedures were performed in accordance with the rules of the Care and Use of Laboratory Animals of the Animal Ethics Committee of Sichuan Agricultural University (Ya'an, China) (approval no. 2013-036). All the methods were carried out in accordance with relevant guidelines and regulations, including any relevant details.

\section{DNA extraction and sequencing}

All samples subjected to DNA extraction were obtained from inside the feces using sterile tweezers to avoid soil contamination with an equal weight of $75 \mathrm{mg}$. Total genomic DNA was extracted from fecal samples using the E.Z.N.A ${ }^{\circledR}$ Stool DNA Kit (Omega Biotechnology, USA) according to manufacturer's instruction, and was stored at $-70{ }^{\circ} \mathrm{C}$ before further analysis. DNA was amplified by using the $515 \mathrm{f} / 806 \mathrm{r}$ primer set (515f: 5'-GTG CCAGCMGCCGCGGTA A-3', 806r: 5'-XXX XXXGGACTACHV GGGTWT CTA AT- $3^{\prime}$ ), which targets the V4 region of the microbial 16S rRNA, with the reverse primer containing a 6-bp error-correcting barcode unique to each sample. PCR reaction was performed using phusion high-fidelity PCR Mastermix (New England Biolabs LTD., Beijing, China) with the following condition: $94{ }^{\circ} \mathrm{C}$ for $3 \mathrm{~min}(1 \mathrm{cycle}), 94{ }^{\circ} \mathrm{C}$ for $45 \mathrm{~s} / 50{ }^{\circ} \mathrm{C}$ for $60 \mathrm{~s} / 72{ }^{\circ} \mathrm{C}$ for $90 \mathrm{~s}$ (35 cycles), and a last step of $72{ }^{\circ} \mathrm{C}$ for $10 \mathrm{~min}$. PCR products were purified by using the QIAquick Gel Extraction Kit (QIAGEN, Dusseldorf, Germany). Sequencing was conducted on an Illumina MiSeq $2 \times 250$ platform according to protocols described by Kozich et al. (2013). Sequencing of partial 16S RNA genes was performed by the Novogene Bioinformatics Technology Co., Ltd. (Beijing, China).

\section{Bioinformatics and statistical analysis}

Sample reads were assembled by using Mothur software package (Schloss et al. 2009), and then quality filtered and demultiplexed in QIIME (quantitative insights into microbial ecology) using default settings (Caporaso et al. 2010). Chimeric sequences were removed by UCHIME (Edgar et al. 2011). Operational Taxonomic Unit (OTUs) were picked using de novo OTU picking protocol with a 97\% similarity threshold and singletons and doubletons 
were discarded in the QIIME software. Taxonomy assignment of OTUs was performed by comparing sequences to the Greengenes database (gg_13_5_otus). Alpha diversity analysis included Shannon index, Chao1, PD whole tree and observed species. Jackknifed beta diversity included both unweighted and weighted Unifrac distances calculated with 10 times of subsampling, and these distances were visualized by Principal Coordinate Analysis (PCoA) (Lozupone and Knight 2005). MannWhitney $U$ test were used for significance test of alpha diversity. Permutational multivariate analysis of variance (PERMANOVA) was used for significance test of beta diversity difference between sample groups. Linear discriminant analysis coupled with effect size (LEfSe) was performed to identify the microbial taxa differentially represented between groups at genus level, which was a robust tool that focused not only on statistical significance but also biological relevance (Segata et al. 2011). The original sequencing data of raw reads were deposited in the sequence read archive of the National Center for Biotechnology (accession nos. PRJNA437450, SRP134182).

\section{Group specific quantitative real-time PCR}

Fecal Microbial population was analyzed using groupspecific primers (Bartosch et al. 2004; Franks et al. 1998; Koike and Kobayashi 2001; Lee et al. 1996; Matsuki et al. 2002, 2004; Rinttila et al. 2004), as listed in Additional file 1: Table S1. qPCR was performed on a Bio-Rad CFX $96^{\text {TM }}$ real-time PCR Detection System with CFX Manager Software version 2.0 (Bio-Rad Laboratories, USA). Each reaction was run in duplicate in a volume of $25 \mu \mathrm{L}$ in low 96-well white PCR plates sealed with optical flat 8-cap strips (Bio-Rad Laboratories, USA). The reaction mixture consisted of $12.5 \mu \mathrm{L} \mathrm{SYBR}^{\circledR}$ Premix Ex Taq ${ }^{\mathrm{TM}}$ II (TaKaRa Biotechnology, China), $1 \mu \mathrm{L}$ of each primer (Invitrogen Life Technologies, China), and $1 \mu \mathrm{L}$ of template DNA of fecal samples (non-template control used water instead). Amplification program included an initial denaturation at $95^{\circ} \mathrm{C}$ for $5 \mathrm{~min}$ followed by 40 cycles of denaturation at $95{ }^{\circ} \mathrm{C}$ for $15 \mathrm{~s}$, primer annealing at the optimal temperatures (Additional file 1: Table S1) for $30 \mathrm{~s}$ and primer extension at $72{ }^{\circ} \mathrm{C}$ for $30 \mathrm{~s}, 1$ cycle of $95^{\circ} \mathrm{C}$ for $1 \mathrm{~min}, 1$ cycle at $55^{\circ} \mathrm{C}$ for $1 \mathrm{~min}$, and a stepwise increase of the temperature from 55 to $95{ }^{\circ} \mathrm{C}$ (at $10 \mathrm{~s} / 0.5{ }^{\circ} \mathrm{C}$ ) to obtain melt curve data. Data were collected at the extension step. Melting curves were checked after amplification and size of PCR products were verified by agarose gels in order to ensure correct amplification results. Standard curves were generated as described by Rinttila et al. (2004) and Schwab and Ganzle (2011).

\section{Results}

\section{Sequencing metadata}

After removing the low quality reads and chimeras, a total of 60,411 chimera-free high quality $16 \mathrm{~S}$ rRNA gene sequences (V4-533-786 bp) were obtained, with an average of $5034.25 \pm 178.35$ sequences per sample, ranging from 4038 to 5321 . These sequences, with average length of $252 \mathrm{bp}$, were assigned to a total of 28,467 operational taxonomic units (OTUs) based on 97\% similarity. Each sample has $1404.25 \pm 308.21$ OTUs on average.

\section{Comparison of microbial community diversity and structures between wild and captive takins}

Microbial community diversity was measured by Shannon index, Chao1, PD whole tree and observed species. All indices were significantly higher in the captive than in the wild takins (Fig. 1, Mann-Whitney $U$ test, $P<0.05$ ), which indicated a higher community diversity (both richness and evenness) of the intestinal microbiota in captive takins. Community structure of the intestinal microbiota also displayed significant separation between wild and captive takins. Both weighted and unweighted UniFrac distances were calculated and Principal Coordinate Analysis (PCoA) was applied to visualize the dissimilarities in community membership (Fig. 2 and Additional file 2: Fig. S1). Based on the membership, microbial communities from captive takins clustered together and separated from those from wild takins along principal coordinate axis. Notably, microbial communities of captive takins clustered tightly, while communities of wild takins were more dispersed. Permutational multivariate analysis of variance (PERMANOVA, $\mathrm{F}=2.72, P=0.005$ ) revealed the differences in community membership between wild and captive takins were statistically significant.

Wild takins possessed similar community structure to captive takins. The microbial communities of takins were mostly dominated by representatives from three phyla: Firmicutes (57.4\%), Bacteroidetes (24.2\%) and Proteobacteria (12.3\%). At family/genus level, Ruminococcaceae, Bacteroidaceae, Acinetobacter, Clostridium, Lachnospiraceae, Rikenellaceae, Bacillus, Comamonas and Spirochaetaceae were dominant. However, the relative abundance of major phyla differed considerably between wild and captive takins. As shown in Fig. 3, the phylum Bacteroidetes contributed significantly more to the intestinal microbiota of captive takins $(28.81 \pm 2.4 \%)$ than wild takins $(19.53 \pm 6.2 \%)$ (Student's $t$ test, $P=0.007)$. The phylum Firmicutes also contributed more to the intestinal microbiota of captive takins $(62.12 \pm 4.1 \%)$ than wild takins $(52.75 \pm 10.9 \%)$, although these trends were not significant (Student's t-test, $\mathrm{P}=0.077$ ). The phylum Proteobacteria contributed significantly more to the 

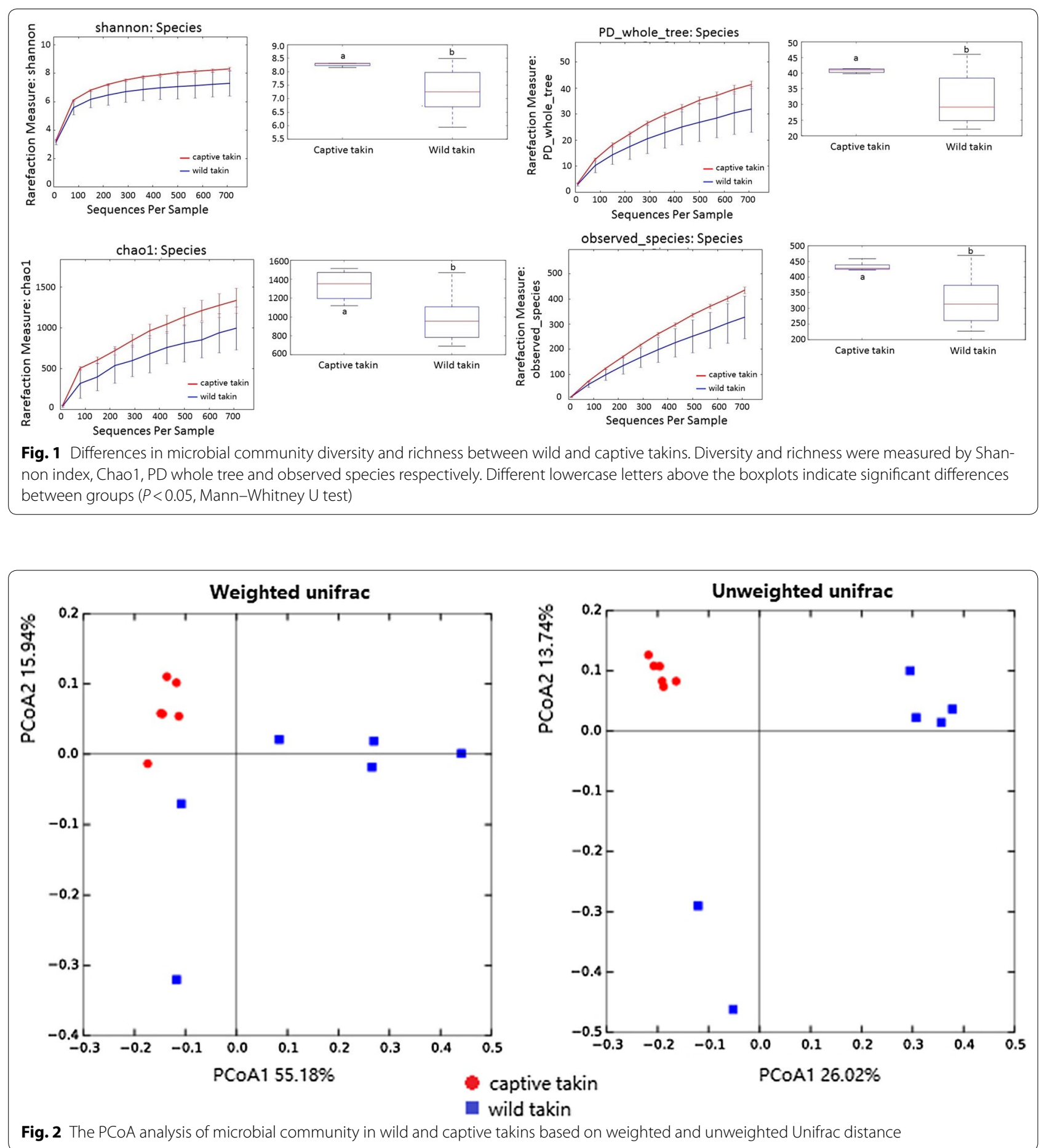

intestinal microbiota of wild takins $(21.57 \pm 19.0 \%)$ than captive takins $(2.9 \pm 0.9 \%)$ (Student's t-test, $P=0.037)$.

The linear discriminant analysis effect size (LEfSe) was employed to identify specific genus that were differentially distributed between wild and captive takins. The results of LEfSe were shown in Fig. 4. A total of 45 genus were differentially represented between the two groups, with 21 more abundant in wild takins (e.g. Acinetobacter, Sphingobacterium, Solibacillus, Lysinibacillus, Pseudomonas, Mycopiana, Adlercreutzia, Stenotrophomonas, YRC 22) and 24 more abundant in captive takins (e.g. Pirellulaceae, Akkermansia, Parabacteroides, 

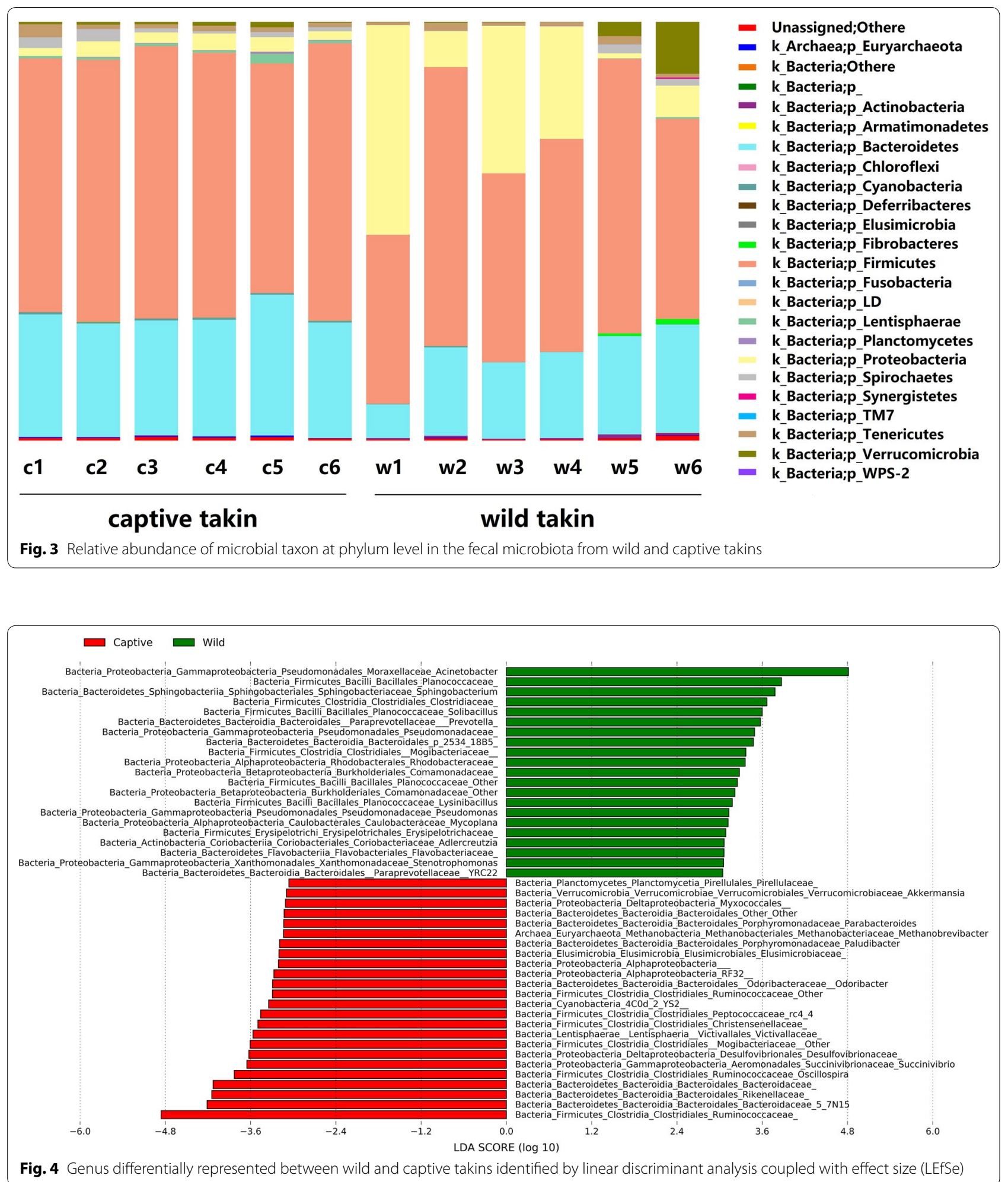
Methanobrevibacter, Paludibacter, Odoribacter, Victivallaceae, Succinivibrio, Oscillospira).

\section{The influence of individual factor on the intestinal microbiota}

Since Illumina Miseq sequencing of partial 16S RNA genes could only provide the relative abundance of microbial taxa, fifteen pairs of group-specific primers were applied to quantify microbial populations in feces of takins. In order to explore the influence of individual factors (age, gender and sampling time) on the intestinal microbiota of takins, predominant microbial populations of three captive takins were monitored for 6 months.

As the quantification results show in Fig. 5, the number of total bacterial gene copies (Eubacteria) in takin feces ranged from 10.98 to $11.35 \log _{10}$ DNA gene copies per gram feces. Blautia coccoides-Eubacterium rectale group represented the majority of the fecal bacterial population, with the average number of $10.67 \pm 0.16 \log _{10}$ DNA gene copies per gram feces. The Bacteroides-PrevotellaPorphyromonas group was also dominant in the feces of takins, which was present at $10.08 \pm 0.17 \log _{10}$ DNA gene copies per gram feces. The number of clostridia in feces of takins was also high. Among the clostridia, the Clostridium clusters IV and XIVa were generally present in equal numbers of approximately $10.09-10.75 \log _{10}$ DNA gene copies per gram feces, even Clostridium cluster I was detected between 7.36 and $9.31 \log _{10}$ DNA gene copies per gram feces. Three presently well-recognized major cellulolytic bacterial species were also detected, Fibrobacter succinogenes was most dominant $(7.77 \pm 0.18$ $\log _{10}$ DNA gene copies per gram feces) among the three species, followed by Ruminococcus albus $(6.93 \pm 0.36$ $\log _{10}$ DNA gene copies per gram feces) and Ruminococcus flavefaciens $\left(6.29 \pm 0.09 \log _{10}\right.$ DNA gene copies per gram feces). Besides, Enterobacteriaceae family was the predominant facultative anaerobe in the feces of takins with a population of $9.6 \pm 0.4 \log _{10}$ DNA gene copies per gram feces. Bifidobacterium spp., Enterococcus spp., Streptococcus spp., Lactobacillus spp. and Fusobacterium spp. also prevailed in the feces of takins $(8.88 \pm 0.25$, $8.31 \pm 0.13,7.73 \pm 0.51,6.49 \pm 0.1$ and $5.77 \pm 0.49 \log _{10}$

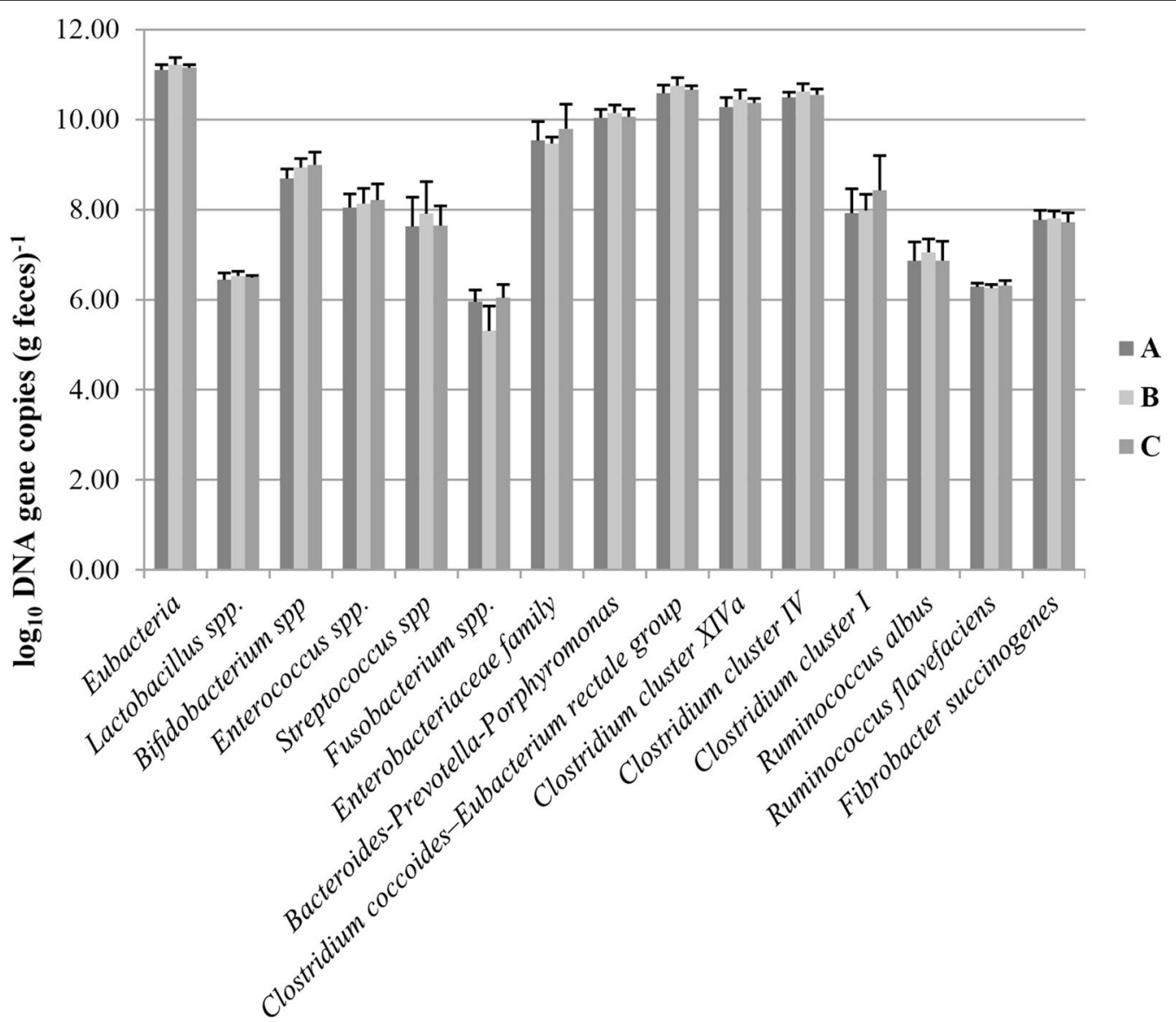

Fig. 5 Average microbial populations in the feces of three takins over all sampling points. A, female takin; B, male takin; C, young male takin 
DNA gene copies per gram feces, respectively). These results were generally consistent with the results of Illumina Miseq sequencing.

From the perspective of the individual, the three takins (subject A, B and C) harbored similar microbial populations. No significant differences were observed in the average numbers of each group/species among the three individuals (Student's t-test, $P>0.05$ ). Differences of each group/species between individuals were all less than 1 $\log _{10}$ DNA gene copies per gram feces. Generally, volatility of microbial populations in the three takins were relatively small during our study period. Significant fluctuations only occurred in the numbers of some microbial taxa like Streptococcus spp., Clostridium cluster I, Fusobacterium spp., Enterobacteriaceae family and Ruminococcus albus, which all fluctuated more than $1 \log _{10}$ DNA gene copies per gram feces during 6-month period (Fig. 6).

\section{Discussion}

To our knowledge, the current work is the first comprehensive study to evaluate the fecal microbiota of the Sichuan takin. In order to effectively study their complex microbial community, Illumina Miseq sequencing and group-specific qPCR had been employed in this study. Based on the results of Illumina Miseq sequencing, the microbial communities of takins were mostly dominated by bacteria belonging to Firmicutes, Bacteroidetes and Proteobacteria, which comprised more than $90 \%$ of the total bacteria. These phyla were also dominant in feces from the cattle, muskoxen and sheep hindgut (De Oliveira et al. 2013; Salgadoflores et al. 2016; Zeng et al. 2017). At family/genus level, Ruminococcaceae, Bacteroidaceae, Acinetobacter, Clostridium, Lachnospiraceae, Rikenellaceae, Bacillus, Comamonas, Oscillospira and Spirochaetaceae constituted the major family/genus. Furet et al. also used qPCR for enumeration of fecal bacteria in human and farm animal (rabbits, goats, horses, pigs, sheep and cows) (Furet et al. 2009). In our study, the same range of populations for the total bacteria and dominant/subdominant bacterial groups like Clostridium, Blautia coccoides and Bacteroides-Prevotella group in sheep and cows were also found in takins. Thus, the fecal microbiota of the takin quite resembled that of cattle, muskoxen and sheep. From the phylogenetic perspective, the takin is in an intermediate position between the cattle and sheep. The takin has similar body size and metabolic requirements to cattle, while their external characteristics are rather similar to sheep. In captivity, the takin even had similar diet and living environment to these animals. All these factors might contribute to captive takin possessing a similar microbial profiles (Demment and Van Soest 1985; Ley et al. 2008b).
Like other herbivores, most dominant bacteria of takins were fiber-digesting bacteria. Ruminococcaceae is one of the most predominant family in the feces of takins. Some Ruminococcaceae played a key role in cellulose degradation (David et al. 2015; Rincon et al. 2001). As members of this family, Ruminococcus flavefaciens, Ruminococcus albus were presently recognized as the major cellulolytic bacterial species found in the rumen (Rincon et al. 2005; Wanapat and Cherdthong 2009). Oscillospira was prevalent in the rumen and hindgut of several herbivores and it is involved in anaerobic fermentation as well (Mackie et al. 2003). Clostridium was another common and diverse genus in takins. In Clostridium, Clostridium cluster XIVa (Blautia coccoides group) and cluster IV (Clostridium leptum subgroup) were generally regarded responsible for the degradation of complex carbohydrates (Burrell et al. 2004). Previous study has also found that putative genes coding two cellulose-digesting enzymes and one hemicellulose-digesting enzyme were recovered in Clostridium group I from intestinal microbes of the panda, which was thought to be an evidence of cellulose metabolism by the giant panda intestinal microbiome (Zhu et al. 2011). The genus Eubacterium was the important constituent of Blautia coccoides-Eubacterium rectale group. Some members affiliated with Eubacterium in the rumen were reported to play the major role in mediating the butyrogenic effect of fermentable dietary carbohydrates (Mitsumori and Minato 1995). In addition, members of the Bacteroides genus were generally considered as the most important group in terms of pectin and lignin degradation, due to their high numbers and nutritional versatility (Akin 1988; Kuritza et al. 1986; Salyers et al. 1977; Weaver et al. 1992). The genus Prevotella is previously classified as member of the genus Bacteroides. These species possess oligosaccharolytic and xylanolytic activities to degrade individual sugars, and also utilize amino acids, and small peptides for their growth, which play a vital role in ruminal carbohydrate and protein fermentation (Marteau et al. 2001). Such as large population of fiber-digesting bacteria would enable the takin a powerful ability to utilize fibrous plant materials as nutrients, as those naturally consumed by wild members. Overall, the microbial community of takins were compatible with their diets structures and metabolic capacities.

To investigate the influence of diets and environmental exposures on the intestinal microbiota, we compared fecal microbiota in takins from Labahe Nature Reserve and Chengdu Zoo. Distinctive microbiotas between wild and captive takins were observed based on community diversity and membership. Compared with wild takins, captive takins had significantly higher community diversity (both richness and evenness). Interestingly, the variation of microbial community structures in 


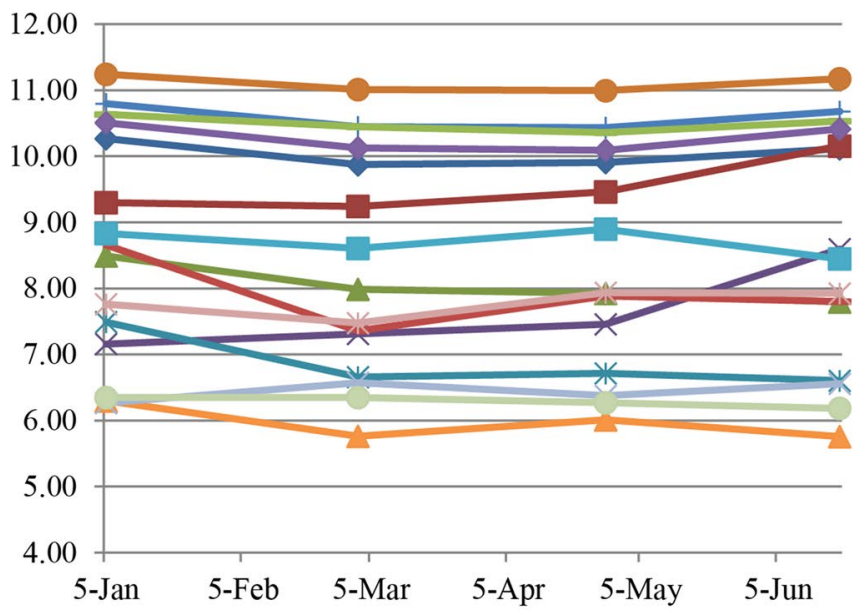

$\longrightarrow$ Bacteroides-Prevotella-Porphyromonas

- Enterobacteriaceae family

Enterococcus spp.

- Ruminococcus albus

$\longrightarrow$ Eubacteria

-Clostridium coccoides-Eubacterium rectale group

Clostridium cluster I

Clostridium cluster IV

$\longrightarrow$ Clostridium cluster XIVa

$=$ Bifidobacterium spp

Fusobacterium spp.

Lactobacillus spp.

-W-Wrobacter succinogenes

$\longrightarrow$ Ruminococcus flavefaciens

subject A
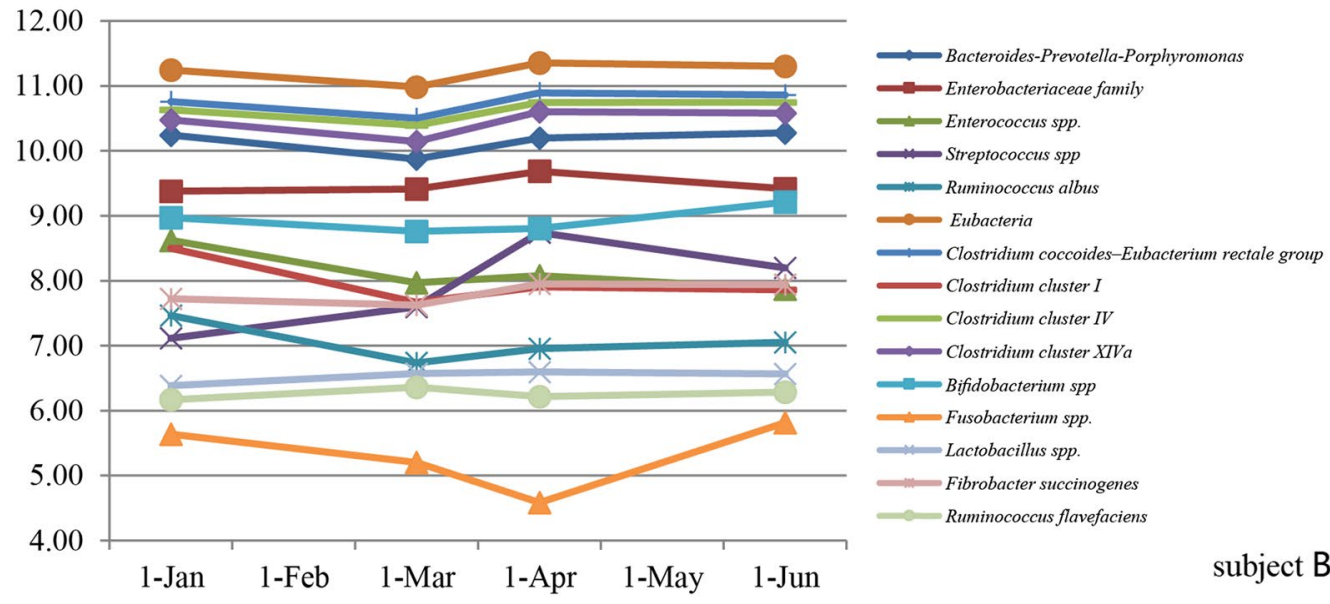

subject $B$
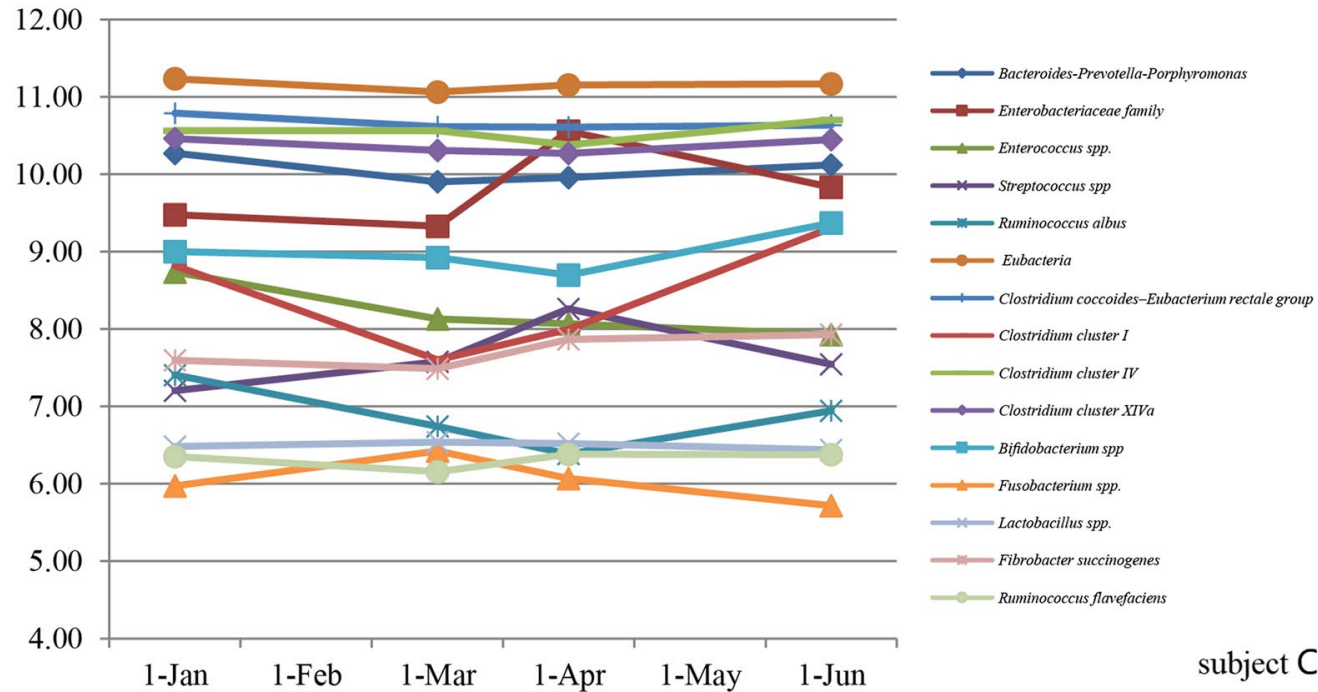

Fig. 6 Microbial populations of three takins over a period of half year 
wild takins was significantly higher than that in captive takins. Diet has been considered as a major driver shaping fecal microbial community composition (Ley et al. 2008a). In captivity, takins are fed a constant and balanced diet, while free-ranging wild takin typically graze on various plants. It could be cautiously speculated that dietary items drove the divergence in the microbiota between wild and captive takins. Meanwhile, random and heterogeneity of diets in wild takins contributed to their large dispersion in microbial community structures. Comparatively, individual factors exerted less influence on microbial community than captivity. As revealed by group-specific qPCR, microbial communities of the three captive takins were relatively similar to each other and stable during our study period. In the few comparable longitudinal studies, microbial communities in the fecal samples of other herbivores (panda, cows and calves) were also stable (Ibekwe et al. 2003; Wei et al. 2007). In contrast, microbial communities present in polar bear feces varied to a larger extent (Schwab and Ganzle 2011). Polar bears harbour simple intestinal physiology and fast digestion times. Therefore, we speculated that relative stability of microbial communities in takins might be caused by their herbivorous diet structure and complex but stable intestinal physiology. Especially for captive takins, they had the same diet composition and the close genetic relationship among individuals. Nevertheless, we still observed an appreciable effect of sampling time on stability of microbial communities in captive takins, which might be caused by seasonal variation in nutritive component of forage grasses and the potential impact of climate on animal physiology.

In summary, we presented the first characterization of the intestinal microbiota in the takins Illumina Miseq sequencing. Distinctive microbiotas between wild and captive takins were observed as a result of their different diet and living environment. Further group-specific qPCR showed that microbial communities of the three takins were relatively similar to each other and stable during our study period, while sampling time had an appreciable effect on microbial communities of the takin. All these results will allow for a better understanding of the putative role of the takin intestinal bacteria in the health and physiological function of the host animal. Of course, the ability to maintain and successfully breed animals threatened with extinction in zoos or protected nature reserves still requires more intimate knowledge of their intestinal microbial ecology and the interrelationships between their native bacteria, diet and nutrient harvest.

\section{Additional files}

Additional file 1: Table S1. Primers used for group-specific quantitative real-time PCR.

Additional file 2: Fig. S1. The PCOA analysis of microbial community in wild and captive takins based on weighted and unweighted Unifrac distance (PC1vsPC3 and PC2vsPC3).

\section{Abbreviations}

qPCR: quantitative real-time PCR; CFU: colony-forming unit; PCoA: Principal Coordinate Analysis; PERMANOVA: permutational multivariate analysis of variance; LEfSe: linear discriminant analysis coupled with effect size.

\section{Authors' contributions}

$\mathrm{HZ}$ designed the experimental program, participated in the examination and drafted the manuscript. DZ conceived of the study, collected the experimental material, and participated in the writing of the zoology section. NW participated in drafting the manuscript. LLN collected and analyzed the raw data. $Y Z$ collected the fecal samples and provided the information of the Sichuan Takin. YZ participated in the GPCR. XQN* (Corresponding author) is responsible for this study, participated in its design and help to draft the manuscript. All authors read and approved the final manuscript.

\section{Author details \\ ${ }^{1}$ Animal Microecology Institute, College of Veterinary, Sichuan Agricultural University, Chengdu 611130, China. ${ }^{2}$ Key Laboratory of Animal Disease and Human Health of Sichuan Province, Chengdu 611130, China. ${ }^{3}$ Col- lege of Bioengineering, Sichuan University of Science and Engineering, Yibin 643000 , China. ${ }^{4}$ Department of Parasitology, College of Veterinary, Sichuan Agricultural University, Chengdu, Sichuan, China. ${ }^{5}$ Chengdu Wildlife Institute, Chengdu Zoo, Chengdu 610081, China.}

\section{Acknowledgements}

Not applicable.

\section{Competing interests}

The authors declare that they have no competing interests.

\section{Availability of data and materials}

The dataset supporting the conclusions of this article is included within this article.

\section{Consent for publication}

Not applicable.

\section{Ethics approval and consent to participate}

All animals were handled in strict accordance with the animal protection law of the People's Republic of China (a draft animal protection law was released on September 18, 2009). All procedures were performed in accordance with the rules of the Care and Use of Laboratory Animals of the Animal Ethics Committee of Sichuan Agricultural University (Ya'an, China) (Approval No. 2013036). All the methods were carried out in accordance with relevant guidelines and regulations, including any relevant details.

\section{Funding}

The present study was supported by the Natural Science Foundation of China (31672318).

\section{Publisher's Note}

Springer Nature remains neutral with regard to jurisdictional claims in published maps and institutional affiliations.

Received: 5 March 2018 Accepted: 18 April 2018

Published online: 27 April 2018 


\section{References}

Akin DE (1988) Biological structure of lignocellulose and its degradation in the rumen. Ani Feed Sci Tech 21:295-310. https://doi. org/10.1016/0377-8401(88)90109-5

Bartosch S, Fite A, Macfarlane GT, Mcmurdo MET (2004) Characterization of bacterial communities in feces from healthy elderly volunteers and hospitalized elderly patients by using real-time PCR and effects of antibiotic treatment on the fecal microbiota. Appl Environ Microb 70:3575-3581. https://doi.org/10.1128/AEM.70.6.3575-3581.2004

Burrell PC, Osullivan C, Song H, Clarke WP, Blackall LL (2004) Identification, detection, and spatial resolution of clostridium populations responsible for cellulose degradation in a methanogenic landfill leachate bioreactor. Appl Environ Microb 70:2414-2419. https://doi.org/10.1128/ AEM.70.4.2414-2419.2004

Caporaso JG, Kuczynski J, Stombaugh J, Bittinger K, Bushman FD, Costello EK, Fierer N, Pena AG, Goodrich JK, Gordon JI (2010) QIIME allows analysis of high-throughput community sequencing data. Nat Methods 7:335-336. https://doi.org/10.1038/nmeth.f.303

David YB, Dassa B, Borovok I, Lamed R, Koropatkin NM, Martens EC, White BA, Bernalierdonadille A, Duncan SH, Flint HJ (2015) Ruminococcal cellulosome systems from rumen to human. Environ Microbiol 17:3407-3426. https://doi.org/10.1111/1462-2920.12868

De Oliveira MNV, Jewell KA, Freitas FS, Benjamin LDA, Totola MR, Borges AC, De Moraes CA, Suen G (2013) Characterizing the microbiota across the gastrointestinal tract of a Brazilian Nelore steer. Vet Microbiol 164:307-314. https://doi.org/10.1016/j.vetmic.2013.02.013

Demment MW, Van Soest PJ (1985) A nutritional explanation for body-size patterns of ruminant and nonruminant herbivores. Am Nat 125:641-672. https://doi.org/10.1086/284369

Eckburg PB, Bik EM, Bernstein CN, Purdom E, Dethlefsen L, Sargent M, Gill SR, Nelson KE, Relman DA (2005) Diversity of the human intestinal microbia flora. Science 308:1635-1638. https://doi.org/10.1126/science.1110591

Edgar RC, Haas BJ, Clemente JC, Quince C, Knight R (2011) UCHIME improves sensitivity and speed of chimera detection. Bioinformatics 27:2194-2200. https://doi.org/10.1093/bioinformatics/btr381

Franks AH, Harmsen HJM, Raangs GC, Jansen GJ, Schut F, Welling GW (1998) Variations of bacterial populations in human feces measured by fluorescent in situ hybridization with group-specific $16 \mathrm{~S}$ rRNA-targeted oligonucleotide probes. Appl Environ Microb 64:3336-3345. https://doi. org/10.12938/bifidus1996.19.79

Furet J, Firmesse O, Gourmelon M, Bridonneau C, Tap J, Mondot S, Dore J, Corthier G (2009) Comparative assessment of human and farm animal faecal microbiota using real-time quantitative PCR. FEMS Microbiol Ecol 68:351-362. https://doi.org/10.1111/j.1574-6941.2009.00671x

Groves P, Shields GF (1997) Cytochrome B sequences suggest convergent evolution of the Asian takin and Arctic muskox. Mol Phylogenet Evolu 8:363-374. https://doi.org/10.1006/mpev.1997.0423

Ibekwe AM, Grieve CM, Lyon SR (2003) Characterization of microbial communities and composition in constructed dairy wetland wastewater effluent. Appl Environ Microb 69:5060-5069. https://doi.org/10.1128/ AEM.69.9.5060-5069.2003

Koike S, Kobayashi Y (2001) Development and use of competitive PCR assays for the rumen cellulolytic bacteria: Fibrobacter succinogenes, Ruminococcus albus and Ruminococcus flavefaciens. FEMS Microbiol Lett 204:361366. https://doi.org/10.1111/j.1574-6968.2001.tb10911x

Kozich JJ, Westcott SL, Baxter NT, Highlander SK, Schloss PD (2013) Development of a dual-index sequencing strategy and curation pipeline for analyzing amplicon sequence data on the MiSeq Illumina sequencing platform. Appl Environ Microb 79:5112-5120. https://doi.org/10.1128/ AEM.01043-13

Kuritza AP, Shaughnessy P, Salyers AA (1986) Enumeration of polysaccharidedegrading Bacteroides species in human feces by using species-specific DNA probes. Appl Environ Microbiol 51:385-390

Lee DH, Zo YG, Kim SJ (1996) Nonradioactive method to study genetic profiles of natural bacterial communities by PCR-single-strand-conformation polymorphism. Appl Environ Microb 62:3112-3120

Ley RE, Hamady M, Lozupone C, Turnbaugh P, Ramey RR, Bircher JS, Schlegel ML, Tucker TA, Schrenzel MD, Knight R (2008a) Evolution of mammals and their gut microbes. Science 320:1647-1651. https://doi.org/10.1126/ science. 1155725
Ley RE, Lozupone CA, Hamady M, Knight R, Gordon Jl (2008b) Worlds within worlds: evolution of the vertebrate gut microbiota. Nat Rev Microbiol 6:776-788. https://doi.org/10.1038/nrmicro1978

Lozupone C, Knight R (2005) UniFrac: a new phylogenetic method for comparing microbial communities. Appl Environ Microb 71:8228-8235. https:// doi.org/10.1128/AEM.71.12.8228-8235.2005

Macfarlane S, Macfarlane GT (2004) Bacterial diversity in the human gut. Adv Appl Microbiol 54:261-289. https://doi.org/10.1016/ S0065-2164(04)54010-8

Mackie Rl, Aminov RI, Hu W, Klieve AV, Ouwerkerk D, Sundset MA, Kamagata $Y$ (2003) Ecology of uncultivated Oscillospira species in the rumen of cattle, sheep, and reindeer as assessed by microscopy and molecular approaches. Appl Environ Microb 69:6808-6815. https://doi.org/10.1128/ AEM.69.11.6808-6815.2003

Marteau P, Pochart P, Doré J, Béramaillet C, Bernalier A, Corthier G (2001) Comparative study of bacterial groups within the human cecal and fecal microbiota. Appl Environ Microb 67:4939-4942. https://doi.org/10.1128/ AEM.67.10.4939-4942.2001

Matsuki T, Watanabe K, Fujimoto J, Miyamoto Y, Takada T, Matsumoto K, Oyaizu H, Tanaka R (2002) Development of $16 \mathrm{~S}$ rRNA-gene-targeted groupspecific primers for the detection and identification of predominant bacteria in human feces. Appl Environ Microb 68:5445-5451. https://doi. org/10.1128/AEM.68.11.5445-5451.2002

Matsuki T, Watanabe K, Fujimoto J, Takada T, Tanaka R (2004) Use of 16 S rRNA gene-targeted group-specific primers for real-time PCR analysis of predominant bacteria in human feces. Appl Environ Microb 70:7220-7228. https://doi.org/10.1128/AEM.70.12.7220-7228.2004

Mitsumori M, Minato H (1995) Distribution of cellulose-binding proteins among the representative strainsof rumen bacteria. J Gen Appl Microbiol 41:297-306. https://doi.org/10.2323/jgam.41.297

Rincon MT, Mccrae SI, Kirby J, Scott KP, Flint HJ (2001) EndB, a multidomain family 44 cellulase from Ruminococcus flavefaciens 17 , binds to cellulose via a novel cellulose-binding module and to another $\mathrm{R}$. flavefaciens protein via a dockerin domain. Appl Environ Microb 67:4426-4431. https:// doi.org/10.1128/AEM.67.10.4426-4431.2001

Rincon MT, Cepeljnik T, Martin JC, Lamed R, Barak Y, Bayer EA, Flint HJ (2005) Unconventional mode of attachment of the Ruminococcus flavefaciens cellulosome to the cell surface. J Bacteriol 187:7569-7578. https://doi. org/10.1128/JB.187.22.7569-7578.2005

Rinttila T, Kassinen A, Malinen E, Krogius L, Palva A (2004) Development of an extensive set of $16 \mathrm{~S}$ rDNA-targeted primers for quantification of pathogenic and indigenous bacteria in faecal samples by real-time PCR. J Appl Microbiol 97:1166-1177. https://doi. org/10.1111/j.1365-2672.2004.02409.x

Russell JB, Rychlik JL (2001) Factors that alter rumen microbial ecology. Science 292:1119-1122. https://doi.org/10.1126/science.1058830

Salgadoflores A, Bockwoldt M, Hagen LH, Pope PB, Sundset MA (2016) A first insight into the faecal microbiota of the high Arctic muskoxen (Ovibos moschatus). Microbial Genomics 2:1-11. https://doi.org/10.1099/ mgen.0.000066

Salyers AA, West SE, Vercellotti JR, Wilkins TD (1977) Fermentation of mucins and plant polysaccharides by anaerobic bacteria from the human colon. Appl Environ Microb 34:529-533

Schloss PD, Westcott SL, Ryabin T, Hall JR, Hartmann M, Hollister EB, Lesniewski RA, Oakley BB, Parks DH, Robinson CJ (2009) Introducing mothur: opensource, platform-independent, community-supported software for describing and comparing microbial communities. Appl Environ Microb 75:7537-7541. https://doi.org/10.1128/AEM.01541-09

Schwab C, Ganzle M (2011) Comparative analysis of fecal microbiota and intestinal microbial metabolic activity in captive polar bears. Can J Microbiol 57:177-185. https://doi.org/10.1139/W10-113

Segata N, Izard J, Waldron L, Gevers D, Miropolsky L, Garrett WS, Huttenhower C (2011) Metagenomic biomarker discovery and explanation. Genome Biol 12:R60. https://doi.org/10.1186/gb-2011-12-6-r60

Stephe AM, Cummings JH (1980) The microbial contribution to human faecal mass. J Med Microbiol 13:45-56. https://doi. org/10.1099/00222615-13-1-45

Wanapat M, Cherdthong A (2009) Use of real-time PCR technique in studying rumen cellulolytic bacteria population as affected by level of roughage in swamp buffalo. Curr Microbiol 58:294-299. https://doi.org/10.1007/ s00284-008-9322-6 
Weaver J, Whitehead TR, Cotta MA, Valentine PC, Salyers AA (1992) Genetic analysis of a locus on the Bacteroides ovatus chromosome which contains xylan utilization genes. Appl Environ Microb 58:2764-2770

Wei G, Lu H, Zhou Z, Xie H, Wang A, Nelson K, Zhao L (2007) The microbial community in the feces of the giant panda (Ailuropoda melanoleuca) as determined by PCR-TGGE profiling and clone library analysis. Microbial Ecol 54:194-202. https://doi.org/10.1007/s00248-007-9225-2

$\mathrm{Wu} \mathrm{H}, \mathrm{Hu} J$ (2001) A comparison in spring and winter habitat selection of Takin, Swtow and Groal and Tangjiahe, Sichuan. Acta Ecol Sin 26:464-469
Zeng Y, Zeng D, Ni X, Zhu H, Jian P, Zhou Y, Xu S, Lin Y, Li Y, Yin Z (2017) Microbial community compositions in the gastrointestinal tract of Chinese Mongolian sheep using Illumina MiSeq sequencing revealed high microbial diversity. Amb Express 7:75. https://doi.org/10.1186/ s13568-017-0378-1

Zhu L, Wu Q, Dai J, Zhang S, Wei F (2011) Evidence of cellulose metabolism by the giant panda gut microbiome. Proc Natl Acad Sci USA 108:1771417719. https://doi.org/10.1073/pnas.1017956108

\section{Submit your manuscript to a SpringerOpen ${ }^{\circ}$ journal and benefit from:}

- Convenient online submission

- Rigorous peer review

- Open access: articles freely available online

- High visibility within the field

- Retaining the copyright to your article

Submit your next manuscript at $\boldsymbol{\nabla}$ springeropen.com 